\title{
The Research on the Injection Interval's Reasonable Water Injection Rate for Multi-Layer Sandstone Oilfield at Ultra-High Water Cut Stage
}

\author{
G.J. SHAN $^{1}$ \\ ${ }^{1}$ Development and research office No.1 of Daqing oilfield exploration and development research \\ institute, Daqing, Heilongjiang, China
}

KEYWORD: Water injection well; Water injection rate of intervals; Water injection efficiency; Grey correlation; Multiple regression

ABSTRACT: The oilfield of Daqing placanticline is a typical multi-layer sandstone oilfield, which water flooding production occupies over $50 \%$ of the total yield of Daqing oilfield, and it has made great contribution to oilfield stable production. For the average water cut is more than $94 \%$ at present, a finer potential tapping for water flooding is needed. In recent years, there is an increasing in interval subdivision of water injection wells, and the average number of intervals is 5 or more in each well. The determination of the injection interval's water injection rate has becoming a problem in that the difference of reservoir nature between intervals is great and the connectivity degree between injection wells and oil wells around is various. The water cut has not risen, meanwhile, the production has not reduced for 5 years in the fine potential tapping demonstration area of the water flooding oilfields in the placanticline, which has been the model of fine water flooding development effect. The reasonable water injection wells are optimized with the demonstration area as the object of analysis and the effect evaluation of oil and water wells as foundation. The rationality of water injection rate of intervals was evaluated based on the water injection efficiency, so as to optimize the reasonable injection interval. The correlation between water injection rate of each interval and the affecting factors was studied using grey correlation and cluster analysis. Then a functional relationship between the water injection rate and the main affecting factors was built using multiple regression. So that a method for the quantitative of water injection rate in intervals is provided. It is convenient to choose the parameters and the method has strong practicality. It has important significance for the extension of water injection optimization technology in the demonstration area.

\section{Introduction}

Ultra-high water cut stage is an important stage of water flooding development. $20 \%$ of the recoverable reserves will be produced at this stage. With the high quality reserves transferring to tertiary recovery year by year, the exploration object is becoming worse at the present stage, the remaining oil distribution is increasingly scattered, and it is more difficult to control water cut and production decline during water flooding. About the optimization of water injection rate, traditional researches are usually aimed at the entire oilfield or block (yang et al. 1998; liu et al. 2015; shan et al. 2013; yang et al. 2013; hu 2012), and the theories are relatively mature. However, theoretical researches aiming at water injection rate of well groups or intervals are not perfect, and domestic and foreign scholars' studies about this are also limited. Heffer KJ, Fox R J and McGill CA introduced kalman filter technique to the judgment of water injection effect, and established kalman filter initial model which can be used to judge the relevance between oil well and water injection well. The model can find out the curve of correlation coefficient of oil and water wells changes with time. Through calculating the correlation coefficient of water injection rate and liquid producing capacity, whether the water injection is effective or not can be judged (Heffer et al. 1995). Alejandro Albertoni, Larry W Lake adopted ridge regression analysis, which declined the parameter estimation error during multiple linear regression, to obtain relatively accurate regression coefficient. It has important significance in adopting multiple linear regression coefficient to interpret the correlation degree between oil and water wells (Alejandro Albertoni \& Larry W Lake 2003). Yin Hongjun et. al. adopted grey correlation analysis method to determine the main factors effecting dividing coefficient and their weight. Quantitative comparison among these factors was realized and an equation about separated layer water injection rate of water injection well was determined (yin et al. 2012). Li Ling, Huang Bingguang et. al. 
put forward an idea that taking water injection well group of water flood field as the research unit and determining the volume of injection allocation using multiple regression method (li et al. 2006). Other scholars also have some researches on water injection rate (zhao et al. 2011; li \& liu. 2006; feng et al. 2011). Field engineers mostly combine the physical property of interval, the connectivity degree of well group, the development index of oil and water wells, and sedimentary facies graph etc. together and give a probable water injection rate of interval. It heavily depends on experiences.

In a word, predecessors mainly focus on the analysis of the relevance between oil well and water injection well and the research of dividing coefficient. However, demonstrations about the rationality of water injection rate are deficient; meanwhile, researches about reasonable water injection rate of interval are less. In this paper, the fine potential tapping demonstration area of the water flooding oilfields is taken as the object of analysis. Reasonable oil and water wells are optimized according to the development index, and a calculation model of the injection interval's reasonable water injection rate, which based on grey comprehensive correlation degree, cluster analysis, and multiple regression, is established. It is convenient to choose the parameters and has strong practicality. And the model provides the basis for field engineers to conduct water injection adjustment at water injection intervals.

\section{Optimization of reasonable water injection wells}

Based on the effect evaluation of oil and water wells, the reasonable water injection wells are optimized.

Oil wells without being stimulated are classified into 4 kinds according to development effect, the classification methods: (1) Evaluation indexes: daily oil production per unit thickness, water cut increasing rate per unit oil production; (2) Take the data of block NDG (normal block) as primary standard, each kind of oil wells occupies about $25 \%$ of total oil wells, and the classification boundary of index is determined. According to the classification boundary, 123 first kind wells with better effect at block BDG are optimized, as shown in Figure 1. For the first kind wells, the water cut increasing rate per one hundred tons oil production is $-0.09 \%$, the average daily oil production per unit thickness is $0.308 \mathrm{t} /(\mathrm{m} \cdot \mathrm{d})$.

According to the connecting relations between oil and water wells, the water wells connecting the first kind wells are determined (obtained by the self-developed software PREP); The reasonable water injection wells are optimized through the index evaluation of water injection. Evaluation indexes: average ratio of water intake layer number and average ratio of water intake thickness. 45 reasonable water injection wells are optimized, as shown in Figure 2.

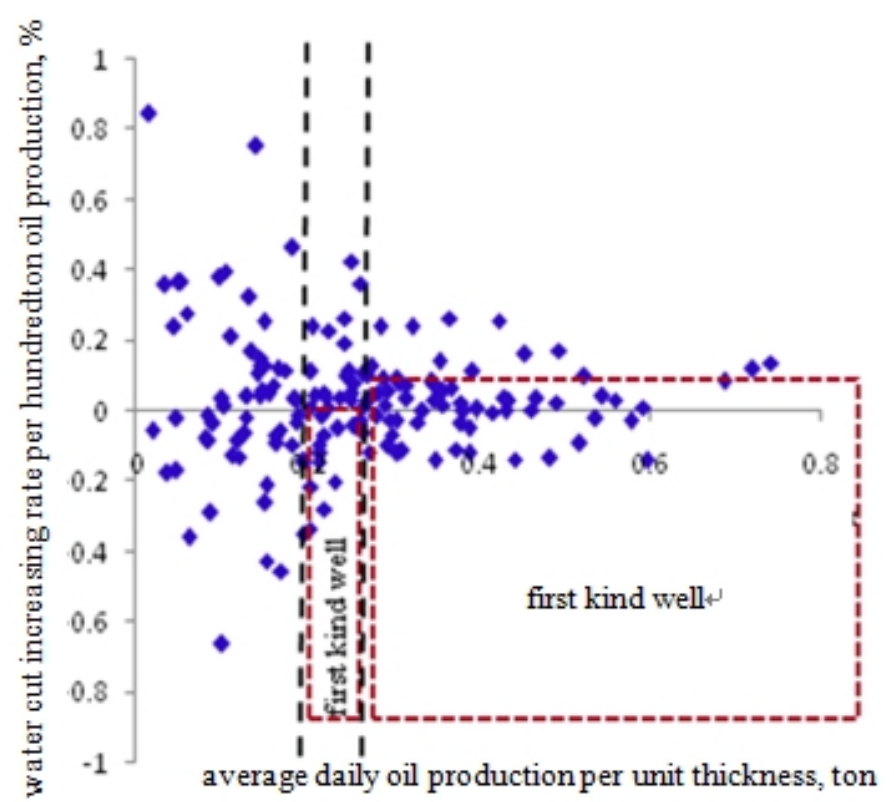

Figure 1. Effect evaluation of oil wells in block BDG 


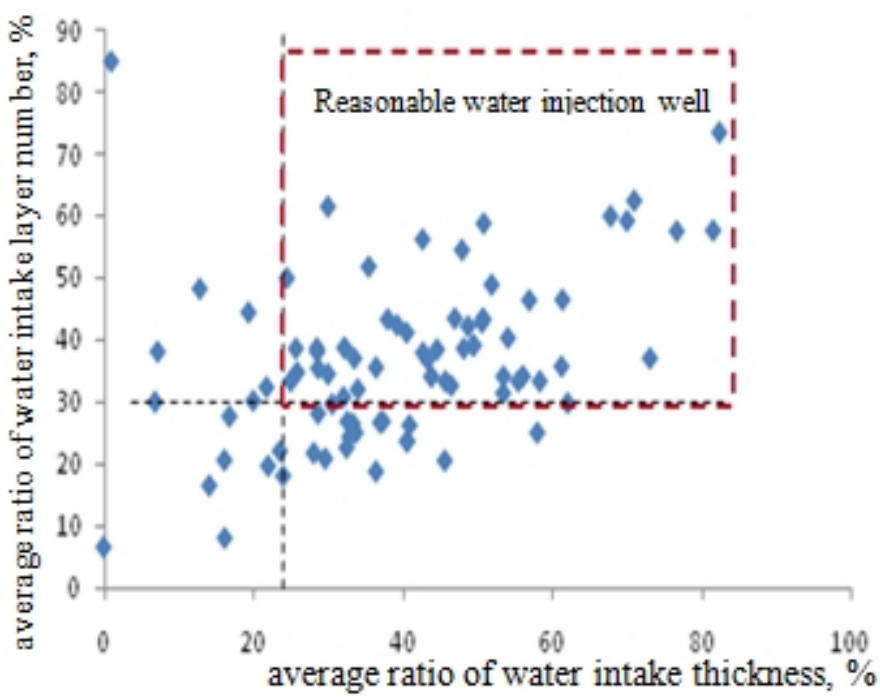

Figure 2. Optimization of reasonable water injection wells in block BDG

\section{Optimization of the reasonable water injection intervals}

\section{Water injection efficiency of intervals}

In order to quantitatively characterize the water injection effect of intervals, the concept of water injection efficiency (Equation 1) is proposed. Its definition is the volume of crude oil which is displaced by unit water injection rate.

$\eta_{w}=\frac{q_{o 1}+q_{o 2}+q_{o 3}+\ldots+q_{o \mathrm{n}}}{q_{w 1}+q_{w 2}+q_{w 3}+\ldots q_{w n}}(\mathrm{n}=1,2,3 \ldots)$

Where $\eta_{w}=$ water injection efficiency of interval, dimensionless; $q_{w n}=$ interval's water injection rate component to the nth connecting oil well, $\mathrm{m}^{3} ; q_{o n}=$ replacing oil rate corresponding to $q_{\mathrm{wn}}, \mathrm{m}^{3}$.

245 intervals of 45 wells are counted. The water injection component of intervals' water injection rate at each direction of the connecting oil well and the corresponding oil production are calculated by the self-developed software PREP (Version 2.0), then the water injection efficiency of all the intervals are calculated. The water injection efficiency of intervals are classified into three types according to the water content level of block, and the reasonable water injection adjustment schemes of the corresponding interval are given, as shown in Table 1.

Table 1 Classification evaluation of water injection efficiency and adjustment scheme of water injection

\begin{tabular}{llll}
\hline $\begin{array}{l}\text { Water injection } \\
\text { efficiency, \% }\end{array}$ & $0 \sim 5$ & $5 \sim 15$ & $15 \sim$ \\
\hline Evaluation grade & Low & Medium & High \\
\hline $\begin{array}{l}\text { Reasonable adjustment } \\
\text { scheme }\end{array}$ & Limit & Succeed & $\begin{array}{l}\text { Streng- } \\
\text { then }\end{array}$ \\
\hline
\end{tabular}

\section{Optimizing Method of reasonable water injection interval}

Compare the historical scheme which has actually happened with reasonable adjustment scheme according to water injection efficiency. If they are identical, it illustrates that the historical scheme is reasonable, and then the interval is a reasonable water injection interval; if not, and then the interval is not a reasonable water injection interval. According to this method, 176 reasonable water injection intervals are optimized. The optimum process is shown in Table 2. 
Table 2 Optimum process of the reasonable injection intervals

\begin{tabular}{|c|c|c|c|c|c|c|}
\hline Well number & Intervals & $\begin{array}{l}\text { Water injection } \\
\text { efficiency }\end{array}$ & $\begin{array}{l}\text { Evaluation } \\
\text { grade }\end{array}$ & Suggested scheme & $\begin{array}{l}\text { Historical } \\
\text { scheme }\end{array}$ & $\begin{array}{l}\text { Reasonable } \\
\text { or not }\end{array}$ \\
\hline G102-36 & G1_11 and above & 0.27 & Low & Limit & Limit & Yes \\
\hline G102-38 & G2 34 to $\mathrm{G} 3 \_3$ & 4.38 & Low & Limit & Succeed & No \\
\hline G101-40 & G2_18 to G2_21 & 7.73 & Medium & Succeed & Succeed & Yes \\
\hline G106-44 & $\mathrm{G} 2 \_14 \_1$ to $\overline{\mathrm{G}} 2 \_27$ & 11.22 & Medium & Succeed or Strengthen & Succeed & Yes \\
\hline G118-CSF42 & $\begin{array}{l}\mathrm{G} 3{ }^{-} 16-1 \text { to } \\
\mathrm{G} 3-19-1\end{array}$ & 17.66 & High & Succeed or Strengthen & Succeed & No \\
\hline G106-F42 & $\mathrm{G} 3{ }^{-} 20$ to $\mathrm{G} 323$ & 27.4 & High & Strengthen & Strengthen & Yes \\
\hline
\end{tabular}

\section{Analysis of the factors affecting the water injection rate of intervals}

\section{Grey comprehensive correlation degree}

Grey comprehensive correlation degree (Equation 2) connects grey absolute correlation degree with grey relative correlation degree. It can not only reflect the similar degree of broken lines of two sequences $X_{0}, X_{1}$, but also reflect the approaching degree of the change rate of the two sequences relative to the starting point.

$$
\rho_{o i}=\theta \varepsilon_{o i}+(1-\theta) r_{o i} \quad(i=1,2,3 \ldots)
$$

Where $\rho_{o i}=$ grey comprehensive correlation coefficient between $X_{0}$ and $X_{\mathrm{i}} ; \varepsilon_{\mathrm{oi}}=$ grey absolute correlation degree between $X_{0}$ and $X_{\mathrm{i}} ; r_{\mathrm{oi}}=$ grey relative correlation degree between $X_{0}$ and $X_{\mathrm{i}} ; \theta=$ ranging from 0 to 1 . If the similarity relation between the absolute amounts is more concerned about, the value can be larger. If the change rate is more concerned about, the value can be smaller. The value is 0.5 in this paper.

\section{Analysis of the influential factors}

According to seepage principle and reservoir engineering principle, also combining the production practice of oilfield, 18 static and dynamic parameters which effect water injection rate of intervals are listed: layer number $\left(X_{1}\right)$, injection pressure $\left(X_{2}\right)$, sandstone thickness $\left(X_{3}\right)$, effective thickness $\left(X_{4}\right)$, reduced effective thickness $\left(X_{5}\right)$, permeability $\left(X_{6}\right)$, formation coefficient $\left(X_{7}\right)$, permeability $\operatorname{ratio}\left(X_{8}\right)$, permeability variation coefficient $\left(X_{9}\right)$, connecting oil well number $\left(X_{10}\right)$, injection-production well spacing $\left(X_{11}\right)$, average flowing pressure of connecting oil well $\left(X_{12}\right)$, average moisture content of connecting oil well $\left(X_{13}\right)$, average sandstone thickness of oil wells $\left(X_{14}\right)$, average effective thickness of oil wells $\left(X_{15}\right)$, average reduced effective thickness of oil wells $\left(X_{16}\right)$, average permeability of oil wells $\left(X_{17}\right)$, average formation coefficient of oil wells $\left(X_{18}\right)$.

Average daily water injection rate of interval is denoted by $X_{0}$, calculate comprehensive correlation coefficient between each $X_{\mathrm{i}}$, as well as $X_{0}$ and $X_{\mathrm{i}}$. According to comprehensive correlation coefficient between each $X_{\mathrm{i}}$ and seepage principle, $X_{\mathrm{i}}$ is classified into five categories (the critical value of correlation coefficient is 0.75 ), the parameter which has the biggest correlation coefficient with $X_{0}$ in each category is used as the main factor affecting water injection rate of interval: $X_{1}, X_{7}, X_{10}, X_{11}$, and $X_{18}$. Correlation coefficients of 5 parameters with $X_{0}$ are shown in Table 3.

Table 3 Comprehensive correlative degree between the main affecting factors of water injection

\begin{tabular}{llllll}
\hline & $X_{1}$ & $X_{7}$ & $X_{10}$ & $X_{11}$ & $X_{18}$ \\
\hline $\begin{array}{l}\text { correlation coeffi- } \\
\text { cient with } X_{0}\end{array}$ & 0.653 & 0.953 & 0.962 & 0.752 & 0.951 \\
\hline
\end{tabular}

\section{Establishment and verification of calculating model of interval's water injection rate}

Based on the theory of multiple regression, the relationship between water injection rate of interval and the main affecting factors is established:

$$
X_{0}=4.035 X_{1}-0.264 X_{7}+0.815 X_{10}-0.019 X_{11}+0.028 X_{18}+24.145
$$

According to the equation, the error between the calculating water injection rate of interval and the actual reasonable water injection rate is less than $20 \%$, shown in Table 4 . It meets the requirement of field engineering, and can be used to guide the water injection adjustment of interval. 
Table 4 Model calculation of water injection rate in intervals

\begin{tabular}{|c|c|c|c|c|c|c|c|}
\hline$X_{1}$ & $X_{7}$ & $X_{10}$ & $X_{11}$ & $X_{18}$ & $\begin{array}{l}\text { Actual water injection } \\
\text { rate, } \mathrm{m}^{3}\end{array}$ & $\begin{array}{l}\text { Calculating water in- } \\
\text { jection rate, } \mathrm{m}^{3}\end{array}$ & Relative error, $\%$ \\
\hline 4 & 2.002 & 5 & 367 & 1.440 & 39 & 37 & 5.4 \\
\hline 8 & 1.131 & 5 & 367 & 2.609 & 62 & 53 & 4.0 \\
\hline 9 & 3.633 & 10 & 452 & 4.946 & 52 & 59 & 13.8 \\
\hline 6 & 1.118 & 5 & 349 & 2.394 & 42 & 46 & 8.5 \\
\hline 3 & 2.647 & 5 & 347 & 3.857 & 33 & 33 & 0.5 \\
\hline 3 & 0.222 & 3 & 236 & 0.363 & 33 & 34 & 3.5 \\
\hline 4 & 1.696 & 5 & 323 & 1.193 & 46 & 38 & 7.8 \\
\hline 4 & 0.754 & 5 & 323 & 2.148 & 42 & 38 & 9.3 \\
\hline 4 & 0.254 & 5 & 323 & 0.383 & 37 & 38 & 3.1 \\
\hline 4 & 0.560 & 6 & 328 & 0.881 & 36 & 39 & 7.8 \\
\hline
\end{tabular}

\section{Conclusions}

The fine potential tapping demonstration area of the water flooding oilfields is taken as the object of analysis, and the reasonable water injection intervals are optimized according to the effect evaluation of oil wells, the effect evaluation of water wells, and water injection efficiency of interval. Then the main affecting factors of interval's water injection rate are analyzed based on the theory of grey correlation.

Based on the theory of multiple regression, the equation of interval's water injection rate is established, and the error meets the requirement of field engineering. It provides the basis for the water injection adjustment of interval. It is convenient to choose the parameters and the method has strong practicality.

The experience of demonstration area is made to be tangible, and it benefits the extension of water injection optimization technology.

\section{REFERENCES}

[1] Alejandro Albertoni \& Larry W Lake 2003. Inferring Interwell Connectivity Only From WellRate Flutuations in Waterfloods. SPE 83381

[2] Feng Bin, Lv Aimin, WangWei, et al. 2011. Approach and application of proration and injection allocation at the middle-late production stages in channel sandstone reservoirs, Oil \& Gas Geology 32(54):934-938.

[3] Heffer K J, Fox R J, McGill C A. 1995. Novel Techniques Shows Links between Reservoir Flow Directionality, Earth Stree, Fault Structure and Geomechanical Changes in Ma-ture Waterfloods. SPE 30711.

[4] Hu Xiaohui 2012. Study and Application of Dimensionless Pore Volume Technology to Calculate the Production and Injection Rate, Offshore Oil 32(2):71-74.

[5] Li Cheng Tong \& Liu Xingquan.2006. Determination of Reasonable Production/Injection Ratio in Sanan Development Area at High Water Cut Stage, Petroleum Geology \& Oil Field Development in Daqing 25(5):54-56.

[6] Li Ling, Huang Bingguang, Tan Xingping, et al. 2006. Injection Allocation Rate Determination of Well Groups Using Multiple Regression Method, Xinjiang Petroleum Geology 27(3):357358.

[7] Liu Huanle, Wu Zexin, Zhang Bo, et al. 2015. Study on the Change of Fluid Production of High Water-cut Stage in Different Producing System, Journal of Southwest Petroleum University: Science \& Technology Edition 37(1):123-127.

[8] Shan Wuyi, Song Kaoping, Geng Wenshuang, et al. 2013. Use Of $\varnothing$ Flinetion To Determine Water Injection Rate, Mathematics In practice And Theory 43(13):121-129.

[9] Yang Guohong, Shang Jianlin, Wang Yong, et al. 2013. Application of Injection- Production Ratio Method Based on Waterflood Type Curves to Optimization of Water Injection Allocation Calculation, Xinjiang Petroleum Geology, 34(1):59-62. 
[10] Yin Hongjun, Zhang Junting, Zhang Huanhuan, et al. 2012. Appling Grey Correlation Analysis Method to Determine the Separated Layer Water Injection, Mathematics In practice And Theory 42(13):95-99.

[11] Yang Yanming, Su Jiandong, Ma Peishen, et al 1998. Reasonable Water Injection Rate Calculation For Water Flooding Reservoirs At Different Water-Cut Stages, Shiyou Kantan Yu Kaifa 25(3): 69-71.

[12]Zhao Chunsen, Li Chenglong, Zhang Dan. 2011. The Research on Influence of Using Numerical Simulation Results to Determine Injection-production Structure on the Development Effect, Science Technology and Engineering 34(11):8577-8580. 